\title{
Mushroom and mushroom-vegetable pastes from wild edible mushrooms
}

\author{
WANDA WOŹNIAK, EUGENIA SOBKOWSKA and ANNA KWIATKOWSKA
}

\begin{abstract}
WOŻNIAK, W., SOBKOWSKA, E. \& KWIATKOWSKA, A. 1978: Mushroom and mushroom-vegetable pastes from wild edible mushrooms. - Karstenia is (suppl.).

In this investigation mushroom and mushroom-vegetable pastes were produced from different edible fungl (Thicholoma equestre, Cantharellus cibarius and Xerocomus badius) in fresh, frozen or dried state, as well as from mushroom mar'c after julce extraction. Sauerkraut, brined cucumbers, tomato concentrate, red pepper, carrots etc. has been used as the components of the mushroom-vegetable pastes. From the long list of different compositions a few were selected for their accepted organoleptic value. The principles of the

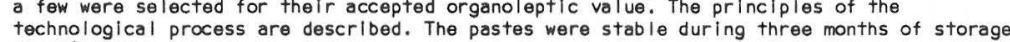
at $12^{\circ} \mathrm{C}$.
\end{abstract}

W. Worniak, E. Sobkowska \&. Kwiatkouska, Institute of Food Technology, Agricultural Academy in Poznañ, ul. Wojska Polskiego 31, Poznañ, Poland.

\section{Material and methods}

Fruit-bodies of Tricholoma equestre (L. ex Fr.) Quél, Cantharellus cibarius Fr. and Xerocomus badius (Fr.) Kuhn were collected from pine woods in western Poland (Miedzychód region) in the autumns of 1973-1976 in 25-40 kg samples and kindly supplied by the company "Las". According to Polish Norm $\frac{\mathrm{PN}-76}{\mathrm{R}-7859}$ the mushrooms were of the first quality class.

Dry weight (at $105^{\circ} \mathrm{C}$ ), titrable acidity,esters (as ethyl acetate) and Kjeldahl $\mathrm{N}$ were measured by standard methods, and protein $\mathrm{N}$ and $\mathrm{NH}_{2}-\mathrm{N}$ according to Bielozierski (1954). The sodium, potassium and calcium contents were estimated in a Carl Zeiss Flamephotometer, model III. Samples were dried, carbonized and finally ashed in a muffle furnace at $550^{\circ} \mathrm{C}$ for $3 \mathrm{~h}$. The redistilled water + $\mathrm{HCl}$ (a few drops) solution of ash was freed from phosphates by using ion exchange resin Reoxin 201. Zinc and iron were determined polarographically using OH 102 Redelkis polarograph. Samples were wet mineralized in a mixture of $\mathrm{HNO}_{3}$ and $\mathrm{H}_{2} \mathrm{SO}_{4}$. For sensory evaluation the five-unit organoleptic method of Tilgner (1957) was used.

The technological process of paste production is shown in Fig. 1. Mushrooms were pulped in a Rietz disintegrator and homogenized in a Frym homogenizer with $0.55 \mathrm{~mm}$ slit. Mushroom marc was obtained after pressing the pulp in the Bucher-Gyuer AC, TPZ-7 layer press of pilot plant scale.

$x$.badius was dried after slicing in air blast dryer at $50-60^{\circ} \mathrm{C}$ during $12 \mathrm{~h}$ to $6 \%$ water content and extracted after crushing in the laboratory Quickfit extractor at $50^{\circ} \mathrm{C}$. The marc after extraction was used for paste production.

\section{Results and discussion}

As stated in the previous paper (Wokniak \& Sobkowska 1978) mushroom juice concentrate production leaves a large amount of marc 30-40\% of raw material weight) containing much of the aromatic and nutritive substances of the fruit-bodies (Table 1). The second grade frozen mushrooms also offer much product that can be processed further into mushroom and mushroom -vegetable pastes (Sobkowska \& Wozniak 1978).

In these investigations different compositions of mushroom and mushroom-vegetable pastes were prepared. Whole fruit-bodies of $T$. equestre were found to be a good material for mushroom paste production

Table 1. Composition of marc of Tricholoma equestre as percent of total content in mushroom

\begin{tabular}{|lcc|}
\hline Compound & Marc from mushrooms \\
\cline { 2 - 3 } & Fresh, recovery\% Frozen, recovery\% \\
\hline Dry weight & 75 & 65 \\
Titrable acids & 58 & 56 \\
Esters & 55 & 51 \\
Total N & $67-75$ & $65-71$ \\
Protein N & $67-73$ & $72-76$ \\
NH $H_{2}$ N & $68-73$ & $45-50$ \\
Ca & 60 & 55 \\
Na & 35 & 30 \\
K & 32 & 25 \\
$\mathrm{Zn}$ & 66 & 63 \\
$\mathrm{Fe}$ & 80 & 70 \\
\hline
\end{tabular}


(disintegration, homogenizing, addition of $10 \%$ of oil fried with onion and/or $2 \%$ of salt, and sterilization, as shown in Fig. 1). For this purpose fresh and frozen mushroom could be used, as well as the marc after juice pressing. In the last mentioned case the organoleptic scores have been about 0.5 unit lower owing to the poorer taste and aroma characteristics (Table 2). The bitter taste makes the problem of paste production from $C$. cibarius more complicated,especial- ly when marc was used. Therefore this mushroom has been found more suitable as material for two-component and mushroom-vegetable pastes than for single mushroom paste production. The situation was similar with $x$. badius, but the limiting factor for single mushroom paste production was the very intensive aroma and rancid taste that caused the low organoleptic evaluation notes (Table 2).

In Tables 2 and 3 some compositions of mush-

Fig. 1. Technological process for paste preparation.

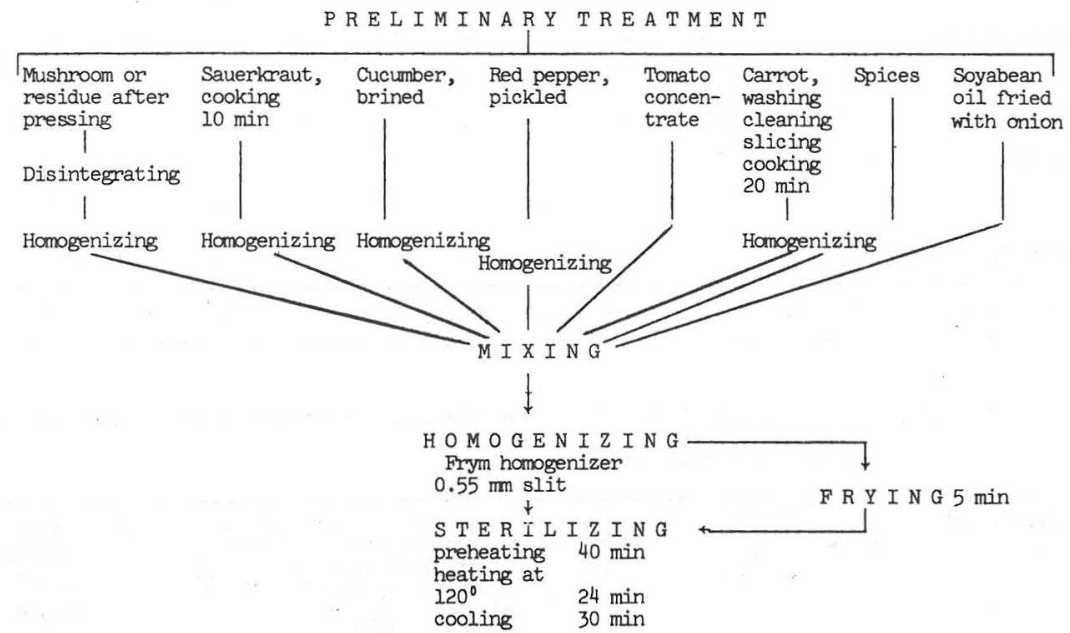

Table 2. Effect of composition on the onganoleptic value of mushroam "and mushroom -vegetable pastes from Tricholoma equestre, Xerocomus badius and Cantharellus cibarius

\begin{tabular}{|c|c|c|c|c|c|c|c|c|c|c|c|}
\hline Component & $\%$ & $\%$ & $\begin{array}{l}\mathrm{C} \\
\%\end{array}$ & o $\mathrm{m}$ & $\mathrm{p}$ & s & $i t$ & i $\%$ & $\begin{array}{l}\mathrm{n} \\
\%\end{array}$ & $\%$ & $\%$ \\
\hline \multicolumn{12}{|l|}{ T. equestre } \\
\hline pulp & 98 & - & - & - & - & - & - & - & - & - & - \\
\hline marc from fresh & - & 98 & - & 56 & 50 & 45 & - & - & 30 & - & - \\
\hline marc, frozen & - & - & 98 & - & - & - & 45 & - & - & - & - \\
\hline $\begin{array}{l}\text { marc, frozen, stored } \\
24 \text { months }\end{array}$ & - & - & - & - & - & - & - & 45 & - & - & - \\
\hline \multicolumn{12}{|l|}{ x. badius } \\
\hline pulp & - & - & - & - & - & - & - & - & 39 & 98 & - \\
\hline $\begin{array}{l}\text { marc after } \\
\text { extraction }\end{array}$ & - & - & - & 24 & - & - & - & - & - & - & - \\
\hline sauerkraut & - & - & - & - & 24 & 17 & 17 & 17 & 6 & - & - \\
\hline red pepper & - & - & - & 7 & - & 21 & 21 & 21 & 6 & - & - \\
\hline cucumber & - & - & - & - & 24 & 15 & 15 & 15 & - & - & - \\
\hline tomato concentrate & - & - & - & - & - & - & - & - & 8 & - & - \\
\hline carrot & - & - & - & 8 & - & - & - & - & 6 & - & - \\
\hline soyabean oil & - & - & - & 3 & - & - & - & - & 3 & - & - \\
\hline salt & 2 & 2 & 2 & 2 & 2 & 2 & 2 & 2 & 2 & 2 & 2 \\
\hline spices & - & - & - & + & - & - & - & - & - & + & + \\
\hline \multicolumn{12}{|l|}{ c. cibarius } \\
\hline $\operatorname{marc}$ & - & - & - & - & - & - & - & - & - & - & 98 \\
\hline Onganoleptic value & 4.0 & 3.6 & 3.4 & 4.1 & 4.2 & 4.6 & 4.5 & 4.2 & 4.1 & 2.8 & 2.0 \\
\hline
\end{tabular}


Table 3. Effect of the composition on the organoleptic value of mushroom marc-vegetable pastes from Tricholoma equestre, Cantharellus cibarius and Xerocomus badius

\begin{tabular}{|c|c|c|c|c|c|c|c|c|c|c|c|c|c|}
\hline \multirow[t]{2}{*}{ Component } & \multicolumn{2}{|c|}{ Tricholoma } & \multicolumn{3}{|c|}{ equestre } & \multicolumn{8}{|c|}{ Cantharel $\{$ us cibarius } \\
\hline & $\%$ & $\%$ & $\%$ & $\%$ & $\%$ & $\%$ & $\%$ & $\%$ & $\%$ & $\%$ & $\%$ & $\%$ & $\%$ \\
\hline \multirow{2}{*}{$\begin{array}{l}\text { Residue after } \\
\text { pressing }\end{array}$} & 1 & 2 & 3 & 4 & 5 & 6 & 7 & 8 & 9 & 10 & 11 & 12 & 13 \\
\hline & 66 & 63 & 50 & 50 & 45 & 80 & 80 & 76 & 67 & 60 & 60 & 49 & 34 \\
\hline Sauerkraut & - & 20 & 6.5 & 24 & - & - & - & - & - & - & - & - & - \\
\hline Red pepper & 16 & 15 & 6.2 & - & 21 & - & - & - & 15 & - & - & 19 & - \\
\hline Cucumber & - & - & 6.5 & 24 & 32 & - & - & - & - & - & - & - & - \\
\hline Tomato concentrate & - & - & - & - & - & - & 10 & 8 & 8 & 19 & - & 10 & 24 \\
\hline Carrot & - & - & 6.3 & & & 12 & - & 10 & - & - & - & - & 34 \\
\hline Soyabean oil & 15 & - & 10 & - & - & 4.5 & 8 & 4 & 10 & - & 10 & 10 & 5 \\
\hline Horse-radish & - & - & 12 & - & - & - & - & - & - & - & - & 8 & - \\
\hline Salt & 2 & 2 & 2 & 2 & 2 & 2 & 2 & 2 & 2 & 2 & 2 & 2 & 2 \\
\hline X. badius pulp & - & - & - & - & - & - & - & - & - & 19 & 28 & - & - \\
\hline Organoleptic value & 4.1 & 4.2 & 4.0 & 3.9 & 4.3 & 2.0 & 4.4 & 4.3 & 3.8 & 4.7 & 4.6 & 2.0 & 4.2 \\
\hline
\end{tabular}

Table 4. Effect of storage at $12^{\circ} \mathrm{C}$ on the chemical and organoleptic evaluation of mushroom-vegetable pastes

\begin{tabular}{|c|c|c|c|c|c|c|c|c|}
\hline \multirow{2}{*}{$\begin{array}{l}\text { Sample of } \\
\text { marc }\end{array}$} & \multirow{2}{*}{$\begin{array}{l}\text { Paste } \\
\text { No. }{ }^{*}\end{array}$} & \multirow{2}{*}{$\begin{array}{l}\text { Storage } \\
\text { time, } \\
\text { months }\end{array}$} & \multicolumn{4}{|c|}{ Content in $100 \mathrm{~g}$ of paste } & \multirow[t]{2}{*}{$\mathrm{pH}$} & \multirow{2}{*}{$\begin{array}{l}\text { Mean } \\
\text { organo- } \\
\text { leptic } \\
\text { score }\end{array}$} \\
\hline & & & $\begin{array}{c}\text { Dry } \\
\text { weight } g\end{array}$ & $\begin{array}{l}\text { Titrable } \\
\text { acidity } \\
\text { ml O.I } \\
\text { n NaOH }\end{array}$ & $\begin{array}{l}\text { Volatile } \\
\text { acid ml } \\
0.1 \mathrm{n} \\
\mathrm{NaOH}\end{array}$ & $\begin{array}{l}\text { Total } \\
\mathrm{N} g\end{array}$ & & \\
\hline \multirow[t]{5}{*}{$\begin{array}{l}\text { Tricholoma } \\
\text { equestre }\end{array}$} & 1 & $\begin{array}{l}0 \\
3\end{array}$ & $\begin{array}{l}19.7 \\
19.7\end{array}$ & $\begin{array}{l}45.0 \\
46.3\end{array}$ & $\begin{array}{l}10.0 \\
11.0\end{array}$ & $\begin{array}{l}1.8 \\
1.8\end{array}$ & $\begin{array}{l}4.9 \\
5.0\end{array}$ & $\begin{array}{l}4.1 \\
4.4\end{array}$ \\
\hline & 2 & $\begin{array}{l}0 \\
3\end{array}$ & $\begin{array}{l}12.1 \\
12.0\end{array}$ & $\begin{array}{l}62.5 \\
65.0\end{array}$ & $\begin{array}{l}16.0 \\
17.0\end{array}$ & $\begin{array}{l}1.9 \\
1.9\end{array}$ & $\begin{array}{l}4.6 \\
4.7\end{array}$ & $\begin{array}{l}4.2 \\
4.2\end{array}$ \\
\hline & 3 & $\begin{array}{l}0 \\
3\end{array}$ & $\begin{array}{l}18.6 \\
18.6\end{array}$ & $\begin{array}{l}55.0 \\
55.0\end{array}$ & $\begin{array}{r}8.5 \\
10.0\end{array}$ & $\begin{array}{l}1.8 \\
1.8\end{array}$ & $\begin{array}{l}4.6 \\
4.7\end{array}$ & $\begin{array}{l}4.0 \\
4.0\end{array}$ \\
\hline & 4 & $\begin{array}{l}0 \\
3\end{array}$ & $\begin{array}{l}10.6 \\
10.7\end{array}$ & $\begin{array}{l}52.5 \\
52.3\end{array}$ & $\begin{array}{r}9.7 \\
10.5\end{array}$ & $\begin{array}{l}1.7 \\
1.7\end{array}$ & $\begin{array}{l}4.5 \\
4.5\end{array}$ & $\begin{array}{l}3.9 \\
4.1\end{array}$ \\
\hline & 5 & $\begin{array}{l}0 \\
3\end{array}$ & $\begin{array}{l}9.9 \\
9.9\end{array}$ & $\begin{array}{l}70.0 \\
70.0\end{array}$ & $\begin{array}{l}24.5 \\
24.5\end{array}$ & $\begin{array}{l}1.3 \\
1.3\end{array}$ & $\begin{array}{l}4.3 \\
4.4\end{array}$ & $\begin{array}{l}4.3 \\
4.3\end{array}$ \\
\hline \multirow[t]{8}{*}{$\begin{array}{l}\text { Contharellus } \\
\text { cibarius }\end{array}$} & 6 & $\begin{array}{l}0 \\
3\end{array}$ & $\begin{array}{l}19.8 \\
19.9\end{array}$ & $\begin{array}{l}25.0 \\
26.3\end{array}$ & $\begin{array}{l}0.5 \\
0.5\end{array}$ & $\begin{array}{l}2.4 \\
2.4\end{array}$ & $\begin{array}{l}4.5 \\
4.5\end{array}$ & $\begin{array}{l}2.0 \\
2.0\end{array}$ \\
\hline & 7 & $\begin{array}{l}0 \\
3\end{array}$ & $\begin{array}{l}23.9 \\
24.0\end{array}$ & $\begin{array}{l}60.0 \\
60.0\end{array}$ & $\begin{array}{l}0.7 \\
0.7\end{array}$ & $\begin{array}{l}3.7 \\
3.7\end{array}$ & $\begin{array}{l}5.8 \\
5.8\end{array}$ & $\begin{array}{l}4.4 \\
4.1\end{array}$ \\
\hline & 8 & $\begin{array}{l}0 \\
3\end{array}$ & $\begin{array}{l}16.6 \\
16.6\end{array}$ & $\begin{array}{l}62.5 \\
62.5\end{array}$ & $\begin{array}{l}1.5 \\
1.5\end{array}$ & $\begin{array}{l}4.2 \\
4.2\end{array}$ & $\begin{array}{l}4.8 \\
4.8\end{array}$ & $\begin{array}{l}4.3 \\
4.3\end{array}$ \\
\hline & 9 & $\begin{array}{l}0 \\
3\end{array}$ & $\begin{array}{l}14.4 \\
14.5\end{array}$ & $\begin{array}{l}50.0 \\
50.0\end{array}$ & 13.5 & $\begin{array}{l}2.6 \\
2.6\end{array}$ & $\begin{array}{l}4.2 \\
4.3\end{array}$ & $\begin{array}{l}3.8 \\
3.9\end{array}$ \\
\hline & 10 & $\begin{array}{l}0 \\
3\end{array}$ & $\begin{array}{l}16.5 \\
16.5\end{array}$ & $\begin{array}{l}33.7 \\
35.0\end{array}$ & $\begin{array}{l}1.0 \\
1.0\end{array}$ & $\begin{array}{l}2.8 \\
2.8\end{array}$ & $\begin{array}{l}5.3 \\
5.4\end{array}$ & $\begin{array}{l}4.7 \\
4.5\end{array}$ \\
\hline & 11 & $\begin{array}{l}0 \\
3\end{array}$ & $\begin{array}{l}16.4 \\
16.3\end{array}$ & $\begin{array}{l}38.7 \\
38.8\end{array}$ & $\begin{array}{l}0.7 \\
1.0\end{array}$ & $\begin{array}{l}3.0 \\
3.0\end{array}$ & $\begin{array}{l}5.4 \\
5.4\end{array}$ & $\begin{array}{l}4.6 \\
4.6\end{array}$ \\
\hline & 12 & $\begin{array}{l}0 \\
3\end{array}$ & $\begin{array}{l}22.6 \\
22.8\end{array}$ & $\begin{array}{l}70.0 \\
71.3\end{array}$ & $\begin{array}{l}15.0 \\
16.0\end{array}$ & $\begin{array}{l}2.5 \\
2.5\end{array}$ & $\begin{array}{l}4.3 \\
4.2\end{array}$ & $\begin{array}{l}2.0 \\
2.0\end{array}$ \\
\hline & 13 & $\begin{array}{l}0 \\
3\end{array}$ & $\begin{array}{l}30.0 \\
30.0\end{array}$ & $\begin{array}{l}85.0 \\
85.5\end{array}$ & $\begin{array}{l}2.0 \\
2.2\end{array}$ & $\begin{array}{l}2.5 \\
2.5\end{array}$ & $\begin{array}{l}4.0 \\
4.0\end{array}$ & $\begin{array}{l}4.2 \\
4.3\end{array}$ \\
\hline
\end{tabular}

${ }^{*}$ As in Table 3. 
room marc-vegetable paste are shown, and Table 4 shows the influence of time of storing at $12^{\circ} \mathrm{C}$ on the chemical and organoleptic characteristics of thè pastes listed in Table 3 .

As can be seen from Tables 2 and 3, sauerkraut, red pepper and cucumber are good components of $T$.

equestre paste; carrot and horse-radish can also be used. Tomato concentrate has been eliminated due to the bad colour effect. Addition of $x$. badius pulp was rather neutral. Paste obtained from the marc of frozen mushrooms, stored for as long as two years, were of good organoleptic value.

In $C$. cibarius paste (Table 3 ) the addition of $x$. badius pulp was beneficial for the taste and aroma characteristics, tomato concentrate has also been a good component giving the paste an attractive colour. The addition of red pepper or, even more, of horse -radish caused reduced notes and disqualification.
The pastes obtained were stable chemically and organoleptically during three months of storage at $10-12^{\circ} \mathrm{C}$.

\section{References}

Bielozierski, A. \& Proskuriakow, N. 1954: Ćwiczenia z biochemii roślin. - p. 241, Warszawa, PWRiL.

Sobkowska, E. \& Wozniak, W. 1978: Combined processing of Tricholoma equestre. - Karstenia 18 (suppl.) (This issue).

Tilgner, D.J. 1957: Analiza organoleptyczna zywnosci. - Warszawa, WPLis.

Woßniak, W. \& Sobkowska, E. 1978: Juice concentrates of edible mushrooms. - Karstenia 18 (suppl.). (This issue). 